\title{
Advancing Symptom Alleviation with Palliative Treatment (ADAPT) trial to improve quality of life: a study protocol for a randomized clinical trial
}

\author{
Bridget A. Graney ${ }^{1,15,16^{*}}$ (D), David H. Au², Anna E. Barón ${ }^{3,4}$, Andrew Cheng ${ }^{2,5}$, Sara A. Combs ${ }^{3,6}$,
} Thomas J. Glorioso ${ }^{14}$, Grady Paden ${ }^{2,7}$, Elizabeth C. Parsons ${ }^{2,8}$, Borsika A. Rabin ${ }^{11}$, Debra P. Ritzwoller ${ }^{12}$, Jessica-Jean Stonecipher ${ }^{13}$, Carolyn Turvey ${ }^{9,10}$, Carolyn H. Welsh ${ }^{1,14}$ and David B. Bekelman ${ }^{3,14}$

\begin{abstract}
Background: People living with chronic heart failure (CHF), chronic obstructive pulmonary disease (COPD), and interstitial lung disease (ILD) suffer impaired quality of life due to burdensome symptoms and depression. The Advancing Symptom Alleviation with Palliative Treatment (ADAPT) trial aims to determine the effect of a multidisciplinary, team-based intervention on quality of life in people with these common diseases.

Methods/design: The ADAPT trial is a two-site, patient-level randomized clinical trial that examines the effectiveness of the ADAPT intervention compared to usual care on patient-reported quality of life at 6 months in veterans with CHF, COPD or ILD with poor quality of life and increased risk for hospitalization or death. The ADAPT intervention involves a multidisciplinary team-a registered nurse, social worker, palliative care specialist, and primary care provider (with access to a pulmonologist and cardiologist) — who meet weekly to make recommendations and write orders for consideration by participants' individual primary care providers. The nurse and social worker interact with participants over six visits to identify and manage a primary bothersome symptom and complete a structured psychosocial intervention and advance care planning. The primary outcome is change in patient-reported quality of life at 6 months as measured by the Functional Assessment of Chronic Illness TherapyGeneral questionnaire. Secondary outcomes at 6 months include change in symptom distress, depression, anxiety, disease-specific quality of life hospitalizations, and advance care planning communication and documentation. Intervention implementation will be assessed using a mixed-methods approach including a qualitative assessment of participants' and intervention personnel experiences and a quantitative assessment of care delivery, resources, and cost.

(Continued on next page)
\end{abstract}

\footnotetext{
* Correspondence: bridget.graney@ucdenver.edu

${ }^{1}$ Division of Pulmonary Sciences and Critical Care Medicine, Department of

Medicine, University of Colorado School of Medicine, Anschutz Medical

Campus, Aurora, CO, USA

${ }^{15}$ University of Colorado Anschutz Medical Campus, Aurora, USA

Full list of author information is available at the end of the article
}

(c) The Author(s). 2019 Open Access This article is distributed under the terms of the Creative Commons Attribution 4.0 International License (http://creativecommons.org/licenses/by/4.0/), which permits unrestricted use, distribution, and reproduction in any medium, provided you give appropriate credit to the original author(s) and the source, provide a link to the Creative Commons license, and indicate if changes were made. The Creative Commons Public Domain Dedication waiver (http://creativecommons.org/publicdomain/zero/1.0/) applies to the data made available in this article, unless otherwise stated. 
(Continued from previous page)

Discussion: The ADAPT trial studies an innovative intervention designed to improve quality of life for veterans with common, burdensome illnesses by targeting key underlying factors — symptoms and depression-that impair quality of life but persist despite disease-specific therapies. Leveraging the skills of affiliate health providers with physician supervision will extend the reach of palliative care and improve quality of life for those with advanced disease within routine outpatient care. The hybrid effectiveness/implementation design of the ADAPT trial will shorten the time to broader dissemination if effective and create avenues for future research.

Trial registration: ClinicalTrials.gov, NCT02713347. Registered March 19, 2016.

Keywords: Heart failure, chronic obstructive pulmonary disease, interstitial lung disease, palliative care, quality of life,

\section{Background}

Despite advances in disease-specific therapies, patients with chronic heart failure (CHF), chronic obstructive pulmonary disease (COPD), and interstitial lung disease (ILD) experience significant symptom burden that contributes to impaired quality of life [1-6]. While these diseases have different pathophysiologic mechanisms, patients experience similar symptoms, including dyspnea at rest and with exertion [7, 8], fatigue [9], pain [10, 11], sleep disturbances, and reduced functional status [12]. Dyspnea and fatigue in particular are pervasive in each of these diseases and are key factors leading to reduced quality of life for patients.

In addition to these symptoms, patients with $\mathrm{CHF}$, COPD, and ILD frequently experience depressive symptoms and many meet clinical criteria for depressive disorders [13-16]. The presence of depressive symptoms independently alters a person's experience and interpretation of symptoms, worsening the severity of each [12, 13, 17]. Furthermore, studies suggest that increasing underlying disease severity is associated with increased severity of depression $[15,18,19]$. This results in everincreasing burden of both disease and symptomatic impairment: as disease progresses, symptoms and depression worsen, further reducing quality of life.

Although the need for disease-specific, symptomtargeted therapies and interventions to improve quality of life and other patient-reported outcome measures is increasingly recognized, few are both efficacious and durable. In lung cancer, a disease with high mortality and significant symptom burden, early palliative care improves quality of life and is associated with a mortality benefit [20, 21]. Due to similarities in bothersome and intrusive symptoms, palliative care may be an effective intervention to improve quality of life in patients with CHF, COPD, and ILD but has not been adequately studied, particularly in the outpatient setting. Currently, palliative care in the inpatient setting, where it is utilized more frequently but when patients are very near the end of life, improves quality of life and decreases costs [22]. Incorporating palliative care management strategies into routine outpatient care may extend the reach and efficacy of palliative care interventions, leading to more sustained improvements in quality of life.

The goal of this randomized clinical trial is to improve quality of life for veterans with CHF, COPD, and ILD through an innovative, multidisciplinary, team-based approach combining disease-specific care for symptoms with palliative symptom management and a psychosocial intervention to treat depression. The ADAPT (Advancing Symptom Alleviation with Palliative Treatment) study aims to evaluate intervention effectiveness and implementation. Understanding implementation (i.e., intervention costs, barriers, and facilitators) will inform necessary changes, identify the potential for dissemination, and advance future research focused on improving quality of life.

\section{Methods/design Conceptual foundation}

The ADAPT study utilizes the conceptual framework depicted in Fig. 1 which is based on integrating elements of Lenz's unpleasant symptom theory [23] into an adaptation of the Wilson and Cleary model of health-related quality of life [24]. Given the relationship between underlying disease severity, symptoms, depression, and impaired quality of life, interventions aimed at improving quality of life must target symptoms and depression concurrently. Therefore, in the ADAPT trial, diseasespecific care is provided in conjunction with symptomfocused care, a psychosocial intervention, and advance care planning and communication by employing a multidisciplinary, team-based approach. By addressing individual facets of advanced chronic disease, including symptoms and depression, we hypothesize improvement in participants' quality of life.

In addition to evaluating effectiveness, the ADAPT study design incorporates a mixed "hybrid effectivenessimplementation" approach [25], evaluating individual intervention components simultaneously with the resources required to implement, maintain, and disseminate the intervention. 


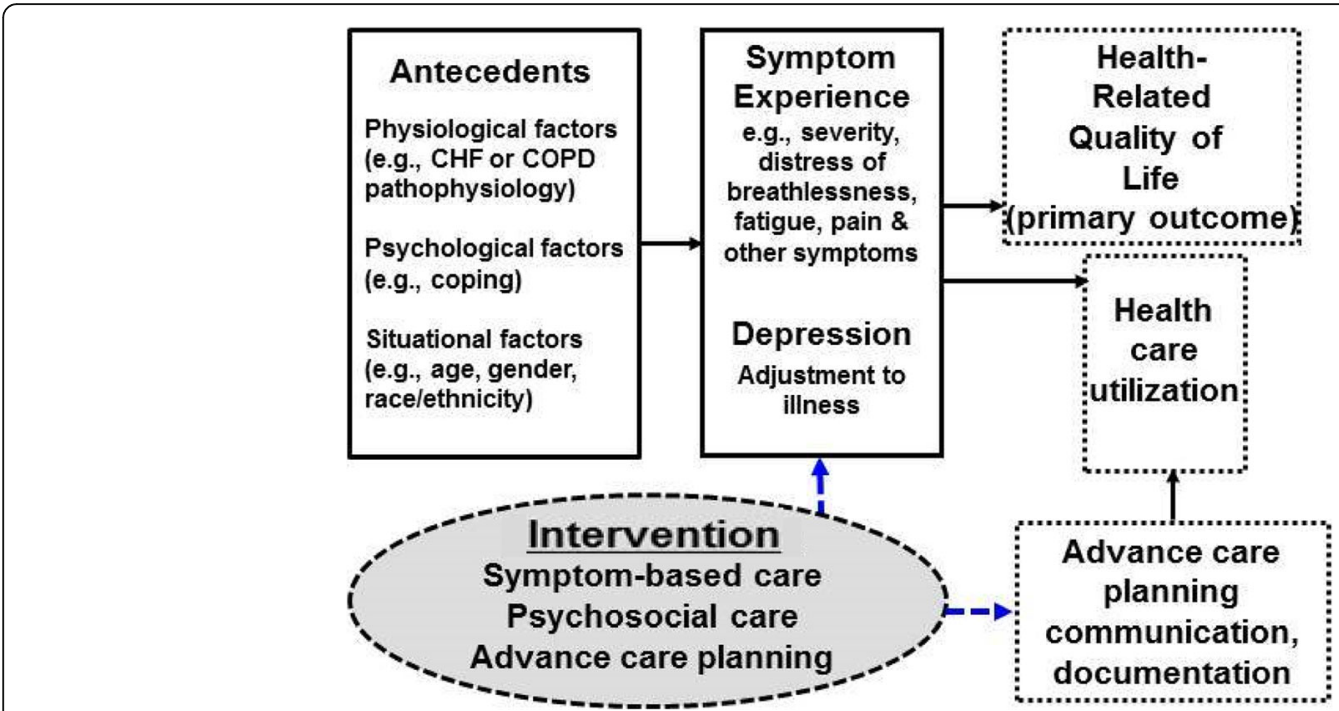

Fig. 1 Conceptual model for the ADAPT trial and proposed intervention effects

\section{Aims and primary hypotheses}

The primary hypothesis is that veterans with $\mathrm{CHF}$, COPD, and ILD randomized to the ADAPT intervention will have improved quality of life at 6 months compared to the control arm of usual care. Secondary hypotheses are that veterans randomized to the intervention will have decreased diseasespecific symptom burden, improvement in depression symptoms, increased advance care planning and communication, and decreased hospitalizations compared to usual care.

Based on the primary hypothesis, the first aim of the study is to determine the effectiveness of the ADAPT intervention on improving quality of life. As the ADAPT intervention targets key contributors to impaired quality of life-symptoms and depression-evaluating these and other secondary outcomes of the study are both clinically meaningful and important to patients, informal (e.g., family) caregivers, and providers.

The secondary aim of the study is to examine implementation of the ADAPT intervention. Utilizing a mixed-methods approach, key stakeholders, including participants, informal caregivers, study personnel, and providers, will provide feedback on the intervention components to assess facilitators and barriers to implementation. Additionally, given that a primary barrier to implementation is the costs and resources required for start-up and maintenance of an intervention [26], these will be evaluated. Incorporating these two facets of intervention testing-effectiveness and implementation testing-can reduce the time delay from innovation discovery to implementation, advance scientific knowledge, and increase policy relevance of clinical research.

\section{Setting}

The study is a randomized clinical trial conducted at two Veterans Health Administration (VA) facilities, the VA Eastern Colorado Health Care System (VA ECHCS) and VA Puget Sound. Each health care system encompasses a tertiary care medical center and seven community-based outpatient clinics.

\section{Study overview}

Veterans with CHF, COPD, or ILD with impaired quality of life who are at increased risk for hospitalization or death are eligible for participation in the study. Enrolled subjects are randomized 1:1 to usual care or the intervention arm (team-based care plus usual care; Fig. 2). Randomization occurs at the patient level with computer generated random block sizes, stratified by study site and disease.

\section{Participants}

The study will enroll 300 veterans with CHF, COPD, or ILD with self-reported poor quality of life and increased risk of hospitalization or death who are able to participate in the intervention. Complete inclusion and exclusion criteria are provided in Table 1.

To identify veterans with increased risk for hospitalizations or death, we use a VA-developed prognostic tool, the Care Assessment Need (CAN) score [27]. The CAN creates a probability estimate of hospital admission or death within one year. Patients with CHF or COPD are potentially eligible to participate in this study if they are in the top 20th percentile of risk for hospitalization or death in the next year. As the CAN score is not validated for use in ILD patients, it will not be used as an eligibility criterion for those with ILD. However, patients 


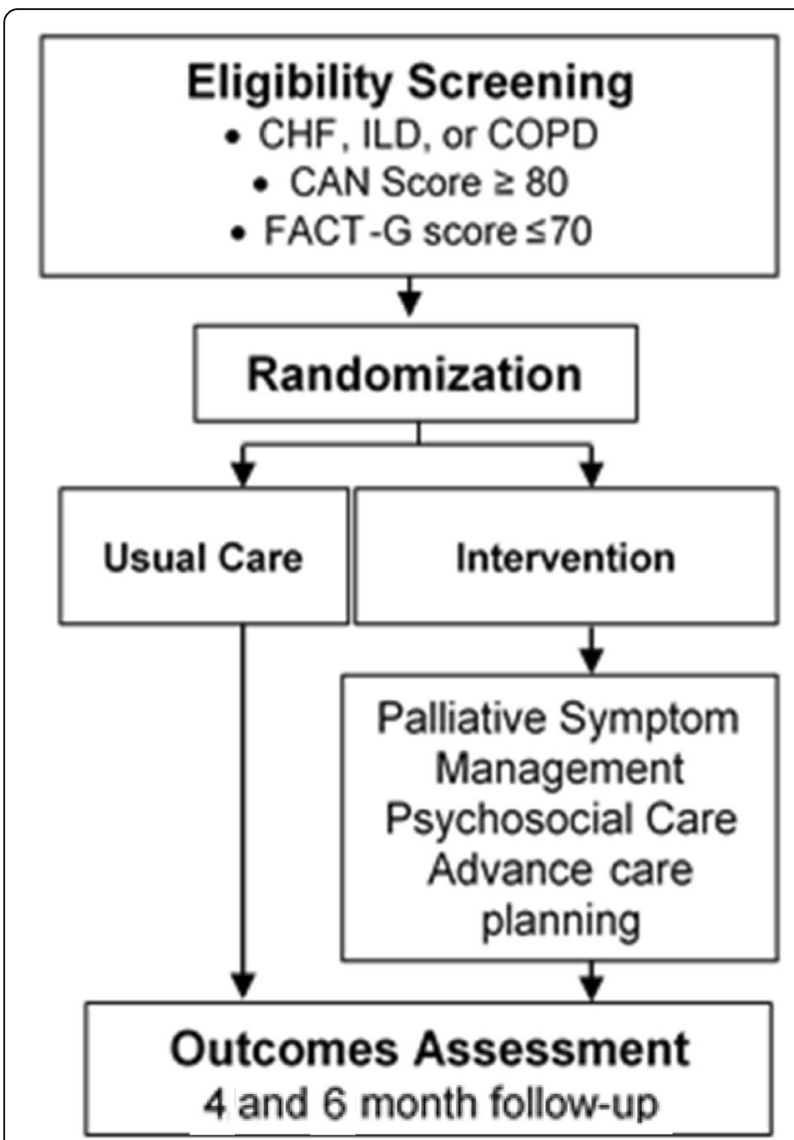

Fig. 2 Flowchart of the ADAPT study

with ILD, particularly fibrotic ILD, have high rates of hospitalization $[28,29]$ with significant associated inhospital or subsequent mortality and are therefore eligible for participation. ILD was approved for addition to the study protocol on May 25, 2018 to expand eligibility criterion.

After meeting diagnostic and at-risk criteria, potential subjects are screened for quality of life using the Functional Assessment of Chronic Illness Therapy-General (FACT-G) questionnaire [30]. A score $\leq 70$ (with lower scores indicating worse quality of life) indicates potential eligibility. This cutoff was chosen because a score $\leq 70$ identifies poor quality of life as validated by declining performance status and increasing disease burden [30].

\section{Recruitment}

To facilitate recruitment and assess eligibility, a HIPAA waiver was granted at both study sites to allow screening of administrative databases and review of medical records. Eligible subjects at both study sites are identified electronically using validated combinations of diagnostic codes for CHF, COPD, or ILD [31, 32]. This generates a list of potential subjects who meet eligibility criteria based on available administrative data. Study personnel then screen individual medical records to confirm eligibility.

After confirmation, patients' primary care providers are contacted to confirm the study team can contact their patients and to explain the study. With primary care provider approval, veterans are mailed letters describing the study and providing contact information for study staff if they are interested in participating. If an eligible veteran does not contact the study team, they are contacted by telephone. We anticipate that it will take 25 months to recruit 300 subjects.

\section{Ethics}

This study has been approved by the Colorado Multiple Institutional Review Board protocol \#15-1891, the VA Puget Sound Multiple Institutional Review Board protocol \#00857 and is registered at ClinicalTrials.gov (NCT02713347). The study is funded by VA HSR\&D IIR 14-346 (Bekelman, Principal Investigator).

\section{Intervention}

The intervention is a multidisciplinary, team-based approach to addressing symptoms and psychosocial needs of participants (Table 2). The team-based approach is based on the evidence-based collaborative care model of health care delivery [33,34]. The intervention personnel include a registered nurse (RN) and Master's level social worker (MSW). They integrate into a larger collaborative care team ("Team") that includes a representative primary care provider (PCP) and palliative care specialist. Specialist support with a cardiologist or pulmonologist is available for the Team for additional management recommendations if needed. Each site has a Team that meets weekly for 30-60 min, integrating palliative symptom management with disease-specific care plans.

The Team is responsible for recording recommendations in a progress note in the electronic medical record, implementing non-pharmacological recommendations, and writing orders for medications or tests that the participants' individual PCPs review and sign at their discretion. This integration creates improved communication between the intervention Team and the PCP and an additional level of safety for participants.

Based on the conceptual model of the study, the intervention includes three pillars: symptom-based care, psychosocial care and advance care planning. Each facet of the ADAPT intervention takes place in collaboration with the Team and participants' individual PCPs. RNs and MSWs interact directly with the participants across several visits as subsequently described.

\section{Symptom-based care}

The intervention personnel make an initial visit either in-person, via phone, or VA telehealth with participants 
Table 1 Eligibility criteria

\begin{tabular}{|c|c|}
\hline Inclusion criteria & Definition \\
\hline $\begin{array}{l}\text { Diagnosis of CHF or COPD within } 2 \text { years prior to enrollment } \\
\text { Diagnosis of ILD within } 2 \text { years prior to enrollment }\end{array}$ & $\begin{array}{l}\text { Hospitalization discharge diagnosis or } \geq 2 \text { outpatient visits } \\
\text { Hospitalization discharge diagnosis or } \geq 1 \text { outpatient diagnosis by a } \\
\text { pulmonologist }{ }^{\mathrm{c}}\end{array}$ \\
\hline $\begin{array}{l}\text { Among those with CHF or COPD, high risk for hospitalization and } \\
\text { death }\end{array}$ & CAN score $\geq 80$ \\
\hline Poor quality of life & FACT-G score $\leq 70$ \\
\hline Symptomatic & $\begin{array}{l}\text { Bothered by at least one of the target symptoms: pain, fatigue, depression, } \\
\text { shortness of breath, trouble sleeping }\end{array}$ \\
\hline $\begin{array}{l}\text { Primary care or other provider who is willing to facilitate } \\
\text { intervention medical recommendations }\end{array}$ & PCP listed in Electronic medical record review or self-report \\
\hline Able to read and understand English & Self-report \\
\hline Consistent access to and able to use a standard telephone & Self-report \\
\hline \multicolumn{2}{|l|}{ Exclusion criteria } \\
\hline Previous diagnosis of dementia & Inpatient or outpatient diagnostic code ${ }^{d}$ \\
\hline Active substance abuse & Electronic medical record review for substance abuse in the previous 6 months \\
\hline Comorbid metastatic cancer & Electronic medical record review \\
\hline Diagnosis of obesity hypoventilation syndrome & Inpatient or outpatient diagnostic code $\mathrm{e}^{\mathrm{e}}$ \\
\hline Nursing home resident & Electronic medical record review or self-report \\
\hline Heart or lung transplant or LVAD & Electronic medical record review or self-report \\
\hline Participation in the intervention arm of the CASA trial ${ }^{f}$ & Electronic medical record review \\
\hline Enrolled in palliative care, hospice, or home-based primary care & Electronic medical record review or self-report \\
\hline Prisoner & Electronic medical record \\
\hline Pregnant & Electronic medical record or self-report \\
\hline
\end{tabular}

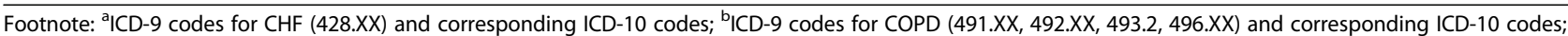
' ICD-9 codes for ILD $(515,516.30,516.31,516.32,516.34,516.37)$ and the corresponding ICD-10 codes; ${ }^{\text {IICD-9 }}$ codes for dementia $(290.0-290.43,291.2,046.1$,

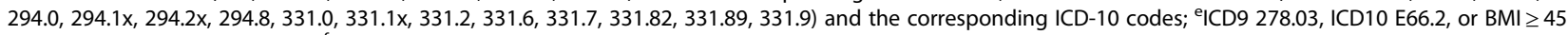
and diagnostic codes for COPD; ${ }^{f}$ Clinicaltrials.gov, NCT01739686. CAN care assessment need, CHF congestive heart failure, CASA collaborative care to alleviate symptoms and adjust to illness, COPD chronic obstructive pulmonary disease, FACT-G Functional Assessment of Cancer Therapy-General, ILD interstitial lung disease, $P C P$ primary care provider

and, if interested, their informal caregivers. Following the initial evaluation with the RN, participants choose a primary symptom-pain, fatigue, mood (including depression or anxiety), shortness of breath, or trouble sleeping-to target for intervention. Participants have the option to change the primary symptom of focus on subsequent visits. After a symptom assessment, the RN follows specific symptom-based algorithms to develop an initial management plan with the Team during a weekly meeting. The RN provides approximately six total visits (two per month) over the study period. For each visit, the $\mathrm{RN}$ is responsible for following-up medical orders and other recommendations, assessing changes in symptoms, communicating with participants' PCPs and other health care providers, and providing education in disease, health care system navigation, and advance care planning to the participants.

\section{Psychosocial care}

The MSW conducts an initial psychosocial assessment [35] and provides six phone-based counseling sessions. The structured counseling was specifically developed and tested in patients with CHF or COPD to improve depression [36] and empower them to discuss issues related to their illness with their care providers. Antidepressant medication can be recommended to

Table 2 Intervention overview

\begin{tabular}{ll}
\hline Intervention component & Personnel \\
\hline $\begin{array}{l}\text { Algorithm-guided symptom management: breathlessness, fatigue, } \\
\text { pain, trouble sleeping }\end{array}$ & Registered nurse (RN) \\
$\begin{array}{ll}\text { Structured psychosocial care, targeting depression and adjustment to } \\
\text { illness; advance care planning }\end{array}$ & Social worker \\
$\begin{array}{ll}\text { Team collaborative care model: } 30-60 \text { min weekly team meetings } & \text { RN, LCSW, palliative and primary care providers. As needed access with } \\
& \text { cardiology and pulmonary specialists }\end{array}$ \\
\hline
\end{tabular}


supplement counseling if the Team determines it is an appropriate, evidence-based treatment recommendation. The MSW assists participants in clarifying health care goals and completing written advance directives.

\section{Advance care planning}

The RN and MSW have a designated visit with participants to discuss care goals and advance care planning. A structured guideline [37] is used to assess participants' understanding of their disease and guide a discussion around goals, concerns, and fears. Informal caregivers if present are invited to participate. The conversation(s) are documented in the electronic medical record and participants will be helped to complete advance directives and state-authorized out-of-hospital order forms if appropriate.

\section{Comparator}

Participants randomized to the control group receive usual care at the discretion of their care providers. They participate in the same number of study visits in addition to completing questionnaires and selfassessments at the same intervals as those in the intervention arm. Usual care will be enhanced by providing participant PCPs with the results of baseline depression surveys if a participant screens positive for depression. Individual providers will then assume responsibility for follow-up of the positive depression screen. Participants in the control arm do not have any limitations on care recommendations or referrals, which may include management by subspecialists, mental health providers, or palliative care at the discretion of their PCPs.

\section{Outcome measures}

The outcome measures and frequency of data collection are summarized in Table 3. Patients self-complete the survey measures and mail surveys to study staff. Surveys are labeled with a study identification number and double-entered by study staff unaware of participant's treatment arm assignment.

\section{Primary outcome}

The primary outcome is patient-reported quality of life. Quality of life is assessed using the Functional Assessment for Chronic illness Therapy-General (FACT-G) questionnaire. The FACT-G is a reliable quality of life questionnaire [38] that is valid in CHF and COPD [39]. It is responsive to specialist palliative care interventions [40] and is correlated with disease severity. Population norms have been established, allowing it to be used across study populations [38]. It measures four subscales that contribute to quality of life: physical, social/family, emotional, and functional well-being. The questionnaire consists of 27 self-report items, each scored on a five-point Likert scale. Individual subscale scores and a total FACT-G score can be determined. Total FACT-G scores range from 0 to 108, with higher scores indicating better quality of life.

\section{Secondary outcomes}

Secondary outcomes were chosen based on domains within the conceptual model of the ADAPT trial.

Symptom experience Symptom distress will be measured using the General Symptom Distress Scale (GSDS) . This is a valid and reliable single item measure of overall symptom distress [41]. It asks, "In general, how distressing are all of your symptoms to you?" It is rated on a numeric rating scale from 0 ("not at all distressing") to 10 ("extremely distressing"). In addition to the overall impact of symptoms, participants will complete questions to assess specific symptoms, including anxiety, fatigue, pain, insomnia, constipation, and numbness/ tingling (Table 3).

Depression Depression will be assessed using the Patient Health Questionnaire-8 (PHQ-8) [42]. The PHQ-8 is a valid, reliable instrument that provides a continuous measure of depressive symptoms. It is both sensitive and specific for a diagnosis of major depressive disorder.

Health-related quality of life The FACT-G is the primary outcome assessment for the ADAPT trial. Participants will also complete subscales of the Quality of Life at the End of Life (QUAL-E) questionnaire, a measure of patient-reported quality of life in advanced illness [43, 44]. It is a valid and reliable instrument utilizing four domains, each scored separately. To assess disease-specific quality of life, study participants will complete one of the following self-report instruments based on their respective underlying diagnosis: the Kansas City Cardiomyopathy Questionnaire (KCCQ-12); the Clinical COPD Questionnaire (CCQ); or the King's Brief Interstitial Lung Disease (K-BILD).

The KCCQ-12 is a valid instrument that measures CHF-specific health status [45]. It is a shortened version of the KCCQ-23, which is a valid, reliable instrument $[46,47]$ that is sensitive to clinical change and predicts hospitalization and mortality. The CCQ is a ten-item self-report instrument that is a valid, reliable, and responsive $[48,49]$ measure of COPD symptoms, functioning, and emotional well-being. The K-BILD is a valid instrument used to assess health status and quality of life for patients with ILD [50]. 
Table 3 Schedule of enrollment, interventions, and assessments

\begin{tabular}{|c|c|c|c|c|c|}
\hline & & Enrollment & Baseline & Month 4 & Month 6 \\
\hline Study Procedure & Measurement & & & & \\
\hline \multicolumn{6}{|l|}{ ENROLLMENT } \\
\hline Eligibility Screen & & $\mathrm{x}$ & & & \\
\hline $\begin{array}{l}\text { Increased Risk of } \\
\text { Hospitalization/Death }\end{array}$ & CAN Score & $\mathrm{x}$ & & & \\
\hline Informed Consent & & $x$ & & & \\
\hline Randomization & & $x$ & & & \\
\hline \multicolumn{6}{|l|}{ INTERVENTIONS } \\
\hline \multirow{2}{*}{\multicolumn{6}{|c|}{$\begin{array}{l}\text { Palliative Symptom } \\
\text { Management }\end{array}$}} \\
\hline & & & & & \\
\hline \multicolumn{6}{|l|}{ Psychosocial Care } \\
\hline \multicolumn{6}{|l|}{ Advance Care Planning } \\
\hline \multicolumn{6}{|l|}{ ASSESSMENTS } \\
\hline Demographics/Study Form & & & $x$ & & \\
\hline $\begin{array}{l}\text { Medications and use of other } \\
\text { medical services }\end{array}$ & Patient report and EMR review & & $\mathrm{x}$ & & $\mathrm{x}$ \\
\hline \multicolumn{6}{|l|}{ Primary Outcome } \\
\hline Health-related quality of life & FACT-G & $x$ & $x$ & $x$ & $x$ \\
\hline \multicolumn{6}{|l|}{ Secondary Outcomes } \\
\hline Overall symptoms & GSDS & & $\mathrm{x}$ & & $x$ \\
\hline \multicolumn{6}{|l|}{ Specific symptoms } \\
\hline Anxiety & GAD-7 & & $x$ & $x$ & $x$ \\
\hline Depression & PHQ-8 & & $x$ & $x$ & $x$ \\
\hline Pain & PEG Scale & & $x$ & & $x$ \\
\hline Fatigue & PROMIS Fatigue & & $\mathrm{x}$ & & $\mathrm{x}$ \\
\hline Insomnia & ISI & & $\mathrm{x}$ & & $\mathrm{x}$ \\
\hline Constipation & Patient report & & $x$ & & $\mathrm{x}$ \\
\hline Numbness/Tingling & Patient report & & $x$ & & $x$ \\
\hline \multicolumn{6}{|l|}{ Advance care planning } \\
\hline $\begin{array}{l}\text { Advance care planning } \\
\text { communication }\end{array}$ & Patient report & & $\mathrm{x}$ & & $x$ \\
\hline $\begin{array}{l}\text { Advance directive } \\
\text { documentation }\end{array}$ & $\begin{array}{l}\text { Presence in the EMR of advance } \\
\text { directive discussion; scanned } \\
\text { advance directive; or medical orders } \\
\text { for life-sustaining treatment }\end{array}$ & & $\mathrm{x}$ & & $x$ \\
\hline Disease-specific health status $^{a}$ & Patient report and EMR review & & $\mathrm{x}$ & & \\
\hline Health-related quality of life & QUAL-E & & $\mathrm{x}$ & & $\mathrm{x}$ \\
\hline Congestive Heart Failure & KCCQ-12 & & $x$ & $x$ & $x$ \\
\hline COPD & CCQ & & $\mathrm{x}$ & $x$ & $\mathrm{x}$ \\
\hline ILD & K-BILD & & $x$ & $x$ & $x$ \\
\hline $\begin{array}{l}\text { Emergency department visits, } \\
\text { hospitalizations, mortality }\end{array}$ & Patient report and EMR review & & & & $x$ \\
\hline Satisfaction & & & $\mathrm{x}$ & & $x$ \\
\hline
\end{tabular}

Adapted from SPIRIT (Standard Protocol Items Recommendations for Interventional Trials) figure. SPIRIT checklist included in Additional file 1.

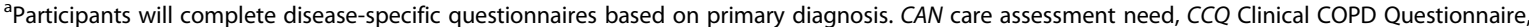
COPD chronic obstructive pulmonary disease, EMR electronic medical record, FACT-G Function Assessment of Cancer Therapy-General, GAD-7 Generalized Anxiety Disorder-7, GSDS General Symptom Distress Scale, ILD interstitial lung disease, ISI Insomnia Severity Index, K-BILD King's Brief Interstitial Lung Disease, KCCQ-12 Kansas City Cardiomyopathy Questionnaire, PEG a three-item scale assessing pain intensity and interference, PHQ-8 Patient Health Questionnaire-8, PROMIS Fatigue patient-reported outcomes measurement information system fatigue scale, QUAL-E Quality of Life at the End of Life

Hospitalizations Patient self-report of hospitalizations will be used as a measure of health care utilization. Medical records from VA and non-VA facilities will be reviewed with permission to validate the patient report.

Advance care planning communication and documentation Readiness to engage in advance care planning is measured using a valid patient reported survey $[51,52]$. Goal concordant care is measured using one question that elicits patient preference for extending life or assuring comfort and another question that assesses patient perception of their current medical care with the same choices [53]. Advance care planning documentation is defined by the presence of a documented advance care planning discussion, scanned advance directive (either a living will or durable power of attorney for health care), or medical orders for lifesustaining treatment within the electronic medical record.

\section{Evaluation of intervention implementation}

Implementation of the intervention will be evaluated using a mixed-methods approach. Using qualitative methods, participants and key study personnel will provide feedback of the implementation process and intervention components utilizing selected Consolidated Framework for Implementation Research (CFIR) domains [54]. Study participants will complete an interview after the 6-month visit with a focus on the value of different intervention personnel, the content and value of the intervention, communication and care coordination, and their perceptions with respect to sustainability of the intervention.

The intervention team will participate in a structured focus group discussion at the completion of the study. The discussion will elicit feedback about what parts of the intervention worked well, might be streamlined, enhanced, or eliminated and views about patient, informal caregiver, and PCP receptivity and responsiveness. 
An intervention database will be used throughout the study to track intervention content and processes. PCPs with patients who complete the intervention will be surveyed to assess their satisfaction and experience with the implementation process. The survey can be completed via email, phone, or in-person to improve total response rates.

We will evaluate the necessary resources to implement and maintain the intervention. We track the time and other resources associated with intervention implementation and maintenance and will subsequently assign cost. The main implementation resource is personnel training. Maintenance costs include personnel time to provide the intervention, including phone calls, patient visits, team meetings, and care coordination with PCPs.

\section{Statistical analysis and sample size}

Data from all participants will be included regardless of level of participation using an intent-to-treat approach. The primary outcome is the difference in FACT-G score at 6 months analyzed as a continuous variable. The sample size was determined to detect a clinically significant difference in the primary outcome of mean FACT-G scores between the intervention and control arms. With an analytic sample size of 115 veterans per arm, we will have $85 \%$ power to detect a moderate effect size of 0.4 (two-sided test, alpha $=0.05$ ). We plan to enroll 300 veterans and anticipate 5\% will die and $15-20 \%$ will have missing outcome data. The minimal clinically important difference on the FACT-G score is $4-6$ points [55], and with a standard deviation of 15 , a Cohen's d effect size of 0.4 will be on the high end of clinical significance.

Because of the anticipated high correlation of baseline FACT-G with follow-up FACT-G $(r>0.5)$, we will include the baseline FACT-G as a precision variable in a linear mixed model of the longitudinal outcome measures. To describe the treatment by disease interaction, we will estimate the treatment effect and its confidence interval within each of the disease groups (CHF, ILD, COPD) using disease-specific health status measures (KCCQ, K-BILD, CCQ) at the 6-month endpoint. In exploratory analyses, we will estimate treatment effect within illness subgroups (CHF, ILD, and COPD) on the primary outcome, and within subgroups of illness, including CHF (preserved vs reduced ejection fraction) and COPD (defined by post-bronchodilator $\mathrm{FEV}_{1} / \mathrm{FVC}$ $<0.70$ on spirometry [56]). Missing data will be reviewed to identify potential patterns and examined to assess how these patterns impact our results. Specifically, we will examine plots of group means over time stratified by the time of the last completed observation to determine if biases are evident due to missing data. When data are missing at random, unbiased results can still be obtained from the maximum likelihood method that will be used in the linear mixed model analysis. To account for the possibility of data missing not at random, sensitivity analyses will be performed using pattern mixture models and results will be presented to assess the impact of missing data on the reported conclusions. Among those who were hospitalized or died, we will examine for differences in intensive care utilization and patterns of care at the end of life. We will also examine for intervention effect on longer-term health care utilization (e.g., hospitalization, intensive care utilization).

The quantitative data on intervention component implementation will be examined using descriptive statistics. For example, for team meetings, the number and type of medical orders written and completed will be summarized. For social worker visits, the median, range, interquartile range, and types of modules completed will be displayed. This type of analysis will show what components of the intervention were actually done. It will characterize the true "dose" and content of the intervention that was provided. This will contribute information about what may have led to intervention success or failure.

The qualitative data on intervention components and processes will be analyzed using a combination of inductive and deductive methods. We will create an evolving set of codes linked to units of text (fragments, sentences, or paragraphs). A qualitative analyst and the research assistant will serve as primary coders for qualitative data, and the PI will review coding and codebooks as they are developed. We will follow a systematic process to enhance coder agreement in assigning codes and a peer debriefing process that requires regular meetings with a qualitative analyst, the PI, and the research assistant to review and refine codes, code definitions, and conceptual boundaries for our analysis. The iterative analysis will begin by using a priori codes based on the CFIR model, supplemented by codes reflecting intervention content and structure and questions used for data collection. Codes will be refined and new codes added as new insights emerge. Through systematic coding we will quickly develop working themes and hypotheses about critical intervention components and processes that will be examined (and inform any minor changes in data collection interview guides/survey). These themes will also describe facilitators and barriers to intervention implementation. We will both audio-record and take detailed notes during all data analysis meetings in order to document proposed codes and code revisions, proposed themes and their descriptions, and other decisions made during these working meetings.

We will use several recommended strategies to enhance the validity and credibility of qualitative findings: 1) structured interview guides administered by welltrained interviewers; 2) coding templates and detailed 
descriptions of codes, coding decisions, and analysis strategies to document all phases of the data analysis (audit trail); and 3) team approaches (at least two analysts) to develop coding templates and independently code subsets of transcripts/notes to determine their agreement and application of codes and code definitions.

In addition to analyzing and summarizing quantitative (implementation tracking database and provider surveys) and qualitative (patient interviews and intervention team focus groups) findings separately, we will also merge findings to draw overarching lessons learned from multiple methods used. In this process, findings will be summarized in a table placing qualitative themes side by side with the quantitative findings to show the extent to which the data converges. Merging these findings will provide "triangulation": findings from each data source will be used to validate and confirm findings from the other data sources. With the combination of qualitative and quantitative data, we expect to provide a more complete explanation of why certain intervention components and processes are more critical than others, as well as facilitators and barriers to the implementation of the intervention.

We will determine resources and cost of the intervention by first calculating the resources (personnel hours or FTE, and other costs) to implement and maintain the intervention during the study. Personnel costs associated with the program will be calculated based on actual VA nurse (and other staff) wage and benefit rates. Total intervention costs and costs per intervention participant at each site will be calculated. Actual salaries and benefits will be used when calculating personnel costs. Second, we will estimate the resources and costs to implement and maintain the program in a variety of VA settings.

Several sensitivity analyses will estimate the range of intervention costs using alternative assumptions for costs that may vary in different implementation contexts. Three sensitivity analyses are planned: 1) variable labor costs with more or less experienced nurses or physicians; 2) variable efficiency of the nurse and social worker in part-time vs full-time intervention roles-this will be done using actual data that reflect the range of time spent per patient early in the study vs later in the study; and 3) variable patient case mix using the range of time spent per patient. In exploratory analyses, we will estimate which patient-level predictors (e.g., cardiac ejection fraction, spirometry, quality of life) are associated with time spent per patient (outcome) using linear regression modeling.

\section{Discussion}

Improving quality of life for patients with chronic disease is an active area of research but few interventions have been found to be effective or scalable. By using a multifaceted approach to target key areas contributing to impaired quality of life, we hope to improve the lives of veterans living with three common chronic diseases, CHF, COPD, and ILD. By using a team-based approach that leverages the successful collaborative care model and the skills of nurses and social workers collaborating with primary care, palliative care, and other specialists, we hope to increase the reach of palliative care. The ADAPT design attempts to integrate symptom-based palliative care interventions into routine outpatient clinical care. If shown to be efficacious, this model could extend the reach of palliative care from primarily inpatient-based care to a routine aspect of primary care for patients with chronic illness with resultant improvements in quality of life.

By utilizing a randomized clinical trial design and examining aspects of implementation, the ADAPT trial will evaluate effectiveness in addition to process and content of the intervention. If the intervention is shown to improve quality of life, having an understanding of the barriers and facilitators of implementation will allow refinement of the ADAPT design for dissemination and integration into other care models. If the intervention is not successful at improving the primary outcome of quality of life, assessment of the intervention will yield valuable information for how to improve the process or content of the implementation for future studies.

\section{Trial status}

The ADAPT study is registered on ClinicalTrials.gov (NCT02713347), registered March 19, 2016. Recruitment began on September 1, 2016 and will be completed on approximately June 30, 2019. Recruitment is ongoing.

\section{Additional file}

Additional file 1: SPIRIT 2013 checklist: Recommended items to address in a clinical trial protocol and related documents*. (DOC $122 \mathrm{~kb}$ )

\section{Abbreviations}

ADAPT: Advancing Symptom Alleviation with Palliative Treatment; CAN: Care Assessment Need; CCQ: Clinical COPD Questionnaire; CHF: Congestive heart failure; COPD: Chronic obstructive pulmonary disease; FACT-G: Functional Assessment of Cancer Therapy_-General; GSDS: General Symptom Distress Scale; ILD: Interstitial lung disease; K-BILD: King's Brief Interstitial Lung Disease; KCCQ-12: Kansas City Cardiomyopathy Questionnaire; PCP: Primary care provider; PHQ-8: Patient Health Questionnaire-8; PRO: Patient-reported outcome; QUAL-E: Quality of Life at the End of Life; VA ECHCS: Veteran's Affairs Eastern Colorado Health Care System

\section{Publisher's Note}

Springer Nature remains neutral with regard to jurisdictional claims in published maps and institutional affiliations.

\section{Acknowledgements}

Dr. Graney would like to acknowledge the contribution of the Reuben M. Cherniak Fellowship Award at National Jewish Health for providing ongoing 
support of her research. We acknowledge the contributions of study staff, including Kelly Blanchard, MSW, LCSW, Veronica Marquez, Brianne Morgan, RN, BSN, PCCN, Brianna Moss, Marilyn Sloan, and Michelle Upham, LICSW.

\section{Authors' contributions}

All authors read and approved the final manuscript.

\section{Funding}

This study is funded by VA HSR\&D IIR 14-346, Bekelman, D. Principal Investigator. Dr. Graney is supported in part by NIH T32 HL007085-44.

\section{Availability of data and materials}

Not applicable.

\section{Ethics approval and consent to participate}

This study has been approved by the Colorado Multiple Institutional Review Board protocol \#15-1891 and the VA Puget Sound Multiple Institutional Review Boards protocol \#00857 and is registered at ClinicalTrials.gov (NCT02713347). All participants will provide informed consent prior to participation.

\section{Consent for publication}

Not applicable.

\section{Competing interests}

The authors declare they have no competing interests.

\section{Author details}

'Division of Pulmonary Sciences and Critical Care Medicine, Department of Medicine, University of Colorado School of Medicine, Anschutz Medical Campus, Aurora, CO, USA. 'Department of Veterans Affairs, Puget Sound Health Care System, Seattle, WA, USA. ${ }^{3}$ Division of General Internal Medicine, Department of Medicine, University of Colorado School of Medicine, Anschutz Medical Campus, Aurora, CO, USA. ${ }^{4}$ Department of Biostatistics and Informatics, Colorado School of Public Health, University of Colorado Anschutz Medical Campus, Aurora, CO, USA. ${ }^{5}$ Division of Cardiology, Department of Medicine, University of Washington School of Medicine, Seattle, WA, USA. 'Division of Renal Diseases and Hypertension, Department of Medicine, University of Colorado School of Medicine, Anschutz Medical Campus, Aurora, CO, USA. ${ }^{7}$ Division of General Internal Medicine, Department of Medicine, University of Washington School of Medicine, Seattle, WA, USA. ${ }^{8}$ Division of Pulmonary, Critical Care and Sleep Medicine, University of Washington, Seattle, WA, USA. 'Department of Psychiatry, University of lowa Carver College of Medicine, lowa City, IA, USA. ${ }^{10}$ Center for Access and Delivery Research and Evaluation, Department of Veterans Affairs, lowa City Health Care System, lowa City, IA, USA. ${ }^{11}$ Department of Family Medicine and Public Health, University of California San Diego School of Medicine, San Diego, CA, USA. ${ }^{12}$ Institute for Health Research, Kaiser Permanente Colorado, Denver, CO, USA. ${ }^{13}$ University Writing Program, University of Florida, Gainesville, FL, USA. ${ }^{14}$ Department of Veterans Affairs, Eastern Colorado Health Care System, Aurora, CO, USA. ${ }^{15}$ University of Colorado Anschutz Medical Campus, Aurora, USA. ${ }^{16}$ National Jewish Health, Anschutz Medical Campus, 12700 East 19th Avenue, Research 2, 9th Floor, Box C272, Aurora, CO 80045, USA.

Received: 22 February 2019 Accepted: 8 May 2019

Published online: 13 June 2019

\section{References}

1. Blinderman CD, Homel P, Billings JA, Portenoy RK, Tennstedt SL. Symptom distress and quality of life in patients with advanced congestive heart failure. J Pain Symptom Manag. 2008;35(6):594-603.

2. Janssen DJ, Franssen FM, Wouters EF, Schols JM, Spruit MA. Impaired health status and care dependency in patients with advanced COPD or chronic heart failure. Qual Life Res. 2011;20(10):1679-88.

3. Blinderman CD, Homel P, Billings JA, Tennstedt S, Portenoy RK. Symptom distress and quality of life in patients with advanced chronic obstructive pulmonary disease. J Pain Symptom Manag. 2009;38(1):115-23.

4. Swigris JJ, Kuschner WG, Jacobs SS, Wilson SR, Gould MK. Health-related quality of life in patients with idiopathic pulmonary fibrosis: a systematic review. Thorax. 2005;60(7):588-94.
5. Yount SE, Beaumont JL, Chen SY, Kaiser K, Wortman K, Van Brunt DL, et al. Health-related quality of life in patients with idiopathic pulmonary fibrosis. Lung. 2016;194(2):227-34.

6. De Vries J, Kessels BL, Drent M. Quality of life of idiopathic pulmonary fibrosis patients. Eur Respir J. 2001;17(5):954-61.

7. Swigris JJ, Streiner DL, Brown KK, Belkin A, Green KE, Wamboldt FS, et al. Assessing exertional dyspnea in patients with idiopathic pulmonary fibrosis. Respir Med. 2014;108(1):181-8.

8. Swigris JJ, Gould MK, Wilson SR. Health-related quality of life among patients with idiopathic pulmonary fibrosis. Chest. 2005;127(1):284-94.

9. Lubin $\mathrm{M}$, Chen H, Elicker B, Jones KD, Collard HR, Lee JS. A comparison of health-related quality of life in idiopathic pulmonary fibrosis and chronic hypersensitivity pneumonitis. Chest. 2014;145(6):1333-8.

10. Bentsen SB, Rustoen T, Miaskowski C. Prevalence and characteristics of pain in patients with chronic obstructive pulmonary disease compared to the Norwegian general population. J Pain. 2011;12(5):539-45.

11. Borge CR, Wahl AK, Moum T. Pain and quality of life with chronic obstructive pulmonary disease. Heart Lung. 2011;40(3):e90-101.

12. Bekelman DB, Havranek EP, Becker DM, Kutner JS, Peterson PN, Wittstein IS, et al. Symptoms, depression, and quality of life in patients with heart failure. J Card Fail. 2007;13(8):643-8.

13. Holland AE, Fiore JF Jr, Bell EC, Goh N, Westall G, Symons K, et al. Dyspnoea and comorbidity contribute to anxiety and depression in interstitial lung disease. Respirology. 2014;19(8):1215-21.

14. Rutledge T, Reis VA, Linke SE, Greenberg BH, Mills PJ. Depression in heart failure a meta-analytic review of prevalence, intervention effects, and associations with clinical outcomes. J Am Coll Cardiol. 2006;48(8):1527-37.

15. Kunik ME, Roundy K, Veazey C, Souchek J, Richardson P, Wray NP, et al. Surprisingly high prevalence of anxiety and depression in chronic breathing disorders. Chest. 2005;127(4):1205-11.

16. Ng TP, Niti M, Fones C, Yap KB, Tan WC. Co-morbid association of depression and COPD: a population-based study. Respir Med. 2009;103(6):895-901.

17. Hilmarsen CW, Wilke S, Engan H, Spruit MA, Rodenburg J, Janssen DJ, et al. Impact of symptoms of anxiety and depression on COPD Assessment Test scores. Eur Respir J. 2014;43(3):898-900.

18. Cully JA, Phillips LL, Kunik ME, Stanley MA, Deswal A. Predicting quality of life in veterans with heart failure: the role of disease severity, depression, and comorbid anxiety. Behav Med. 2010;36(2):70-6.

19. Lacasse Y, Rousseau L, Maltais F. Prevalence of depressive symptoms and depression in patients with severe oxygen-dependent chronic obstructive pulmonary disease. J Cardpulm Rehabil. 2001;21(2):80-6.

20. Temel JS, Greer JA, Muzikansky A, Gallagher ER, Admane S, Jackson VA, et al. Early palliative care for patients with metastatic non-small-cell lung cancer. N Engl J Med. 2010;363(8):733-42.

21. Movsas B, Moughan J, Sarna L, Langer C, Werner-Wasik M, Nicolaou N, et al. Quality of life supersedes the classic prognosticators for long-term survival in locally advanced non-small-cell lung cancer: an analysis of RTOG 9801. J Clin Oncol. 2009;27(34):5816-22.

22. Gade G, Venohr I, Conner D, McGrady K, Beane J, Richardson RH, et al. Impact of an inpatient palliative care team: a randomized control trial. J Palliat Med. 2008;11(2):180-90.

23. Lenz ER, Pugh LC, Milligan RA, Gift A, Suppe F. The middle-range theory of unpleasant symptoms: an update. ANS Adv Nurs Sci. 1997;19(3):14-27.

24. Wilson IB, Cleary PD. Linking clinical variables with health-related quality of life. A conceptual model of patient outcomes. JAMA. 1995;273(1):59-65.

25. Curran GM, Bauer M, Mittman B, Pyne JM, Stetler C. Effectivenessimplementation hybrid designs: combining elements of clinical effectiveness and implementation research to enhance public health impact. Med Care. 2012;50(3):217-26.

26. Jones Rhodes WC, Ritzwoller DP, Glasgow RE. Stakeholder perspectives on costs and resource expenditures: tools for addressing economic issues most relevant to patients, providers, and clinics. Transl Behav Med. 2018;8(5):675-82.

27. Wang L, Porter B, Maynard C, Evans G, Bryson C, Sun H, et al. Predicting risk of hospitalization or death among patients receiving primary care in the Veterans Health Administration. Med Care. 2013;51(4):368-73.

28. Brown AW, Fischer CP, Shlobin OA, Buhr RG, Ahmad S, Weir NA, et al. Outcomes after hospitalization in idiopathic pulmonary fibrosis: a cohort study. Chest. 2015;147(1):173-9.

29. Moua T, Westerly BD, Dulohery MM, Daniels CE, Ryu JH, Lim KG. Patients with fibrotic interstitial lung disease hospitalized for acute respiratory worsening: a large cohort analysis. Chest. 2016;149(5):1205-14. 
30. Pearman T, Yanez B, Peipert J, Wortman K, Beaumont J, Cella D. Ambulatory cancer and US general population reference values and cutoff scores for the functional assessment of cancer therapy. Cancer. 2014;120(18):2902-9.

31. Go AS, Yang J, Ackerson LM, Lepper K, Robbins S, Massie BM, et al. Hemoglobin level, chronic kidney disease, and the risks of death and hospitalization in adults with chronic heart failure: the Anemia in Chronic Heart Failure: Outcomes and Resource Utilization (ANCHOR) Study. Circulation. 2006;113(23):2713-23.

32. Cooke CR, Joo MJ, Anderson SM, Lee TA, Udris EM, Johnson E, et al. The validity of using ICD-9 codes and pharmacy records to identify patients with chronic obstructive pulmonary disease. BMC Health Serv Res. 2011;11:37.

33. Von Korff M, Gruman J, Schaefer J, Curry SJ, Wagner EH. Collaborative management of chronic illness. Ann Intern Med. 1997;127(12):1097-102.

34. Katon WJ, Lin EH, Von Korff M, Ciechanowski P, Ludman EJ, Young B, et al. Collaborative care for patients with depression and chronic illnesses. N Engl J Med. 2010;363(27):2611-20.

35. Workers NAOS. NASW Standards for Palliative \& End of Life Care Washington, DC: NASW Press; 2004.

36. Turvey $\mathrm{CL}$, Klein DM. Remission from depression comorbid with chronic illness and physical impairment. Am J Psychiatry. 2008;165(5):569-74.

37. Bekelman DB, Johnson-Koenke R, Ahluwalia SC, Walling AM, Peterson J, Sudore RL. Development and feasibility of a structured goals of care communication guide. J Palliat Med. 2017;20(9):1004-12.

38. Webster K, Cella D, Yost K. The Functional Assessment of Chronic Illness Therapy (FACIT) measurement system: properties, applications, and interpretation. Health Qual Life Outcomes. 2003;1:79.

39. Steinhauser KE, Arnold RM, Olsen MK, Lindquist J, Hays J, Wood LL, et al Comparing three life-limiting diseases: does diagnosis matter or is sick, sick? J Pain Symptom Manag. 2011;42(3):331-41.

40. Zimmermann C, Swami N, Krzyzanowska M, Hannon B, Leighl N, Oza A, et al. Early palliative care for patients with advanced cancer: a clusterrandomised controlled trial. Lancet. 2014;383(9930):1721-30.

41. Badger TA, Segrin C, Meek P. Development and validation of an instrument for rapidly assessing symptoms: the general symptom distress scale. J Pain Symptom Manag. 2011;41(3):535-48.

42. Kroenke K, Strine TW, Spitzer RL, Williams JB, Berry JT, Mokdad AH. The PHQ8 as a measure of current depression in the general population. J Affect Disord. 2009:114(1-3):163-73

43. Steinhauser KE, Clipp EC, Bosworth HB, McNeilly M, Christakis NA, Voils Cl, et al. Measuring quality of life at the end of life: validation of the QUAL-E. Palliat Support Care. 2004;2(1):3-14

44. Lo C, Burman D, Swami N, Gagliese L, Rodin G, Zimmermann C. Validation of the QUAL-EC for assessing quality of life in patients with advanced cancer. Eur J Cancer. 2011:47(4):554-60.

45. Spertus JA, Jones PG. Development and validation of a short version of the Kansas City Cardiomyopathy Questionnaire. Circ Cardiovasc Qual Outcomes. 2015;8(5):469-76.

46. Spertus J, Peterson E, Conard MW, Heidenreich PA, Krumholz HM, Jones P, et al. Monitoring clinical changes in patients with heart failure: a comparison of methods. Am Heart J. 2005;150(4):707-15.

47. Heidenreich PA, Spertus JA, Jones PG, Weintraub WS, Rumsfeld JS, Rathore SS, et al. Health status identifies heart failure outpatients at risk for hospitalization or death. J Am Coll Cardiol. 2006;47(4):752-6.

48. van der Molen T, Willemse BW, Schokker S, ten Hacken NH, Postma DS, Juniper EF. Development, validity and responsiveness of the Clinical COPD Questionnaire. Health Qual Life Outcomes. 2003;1:13.

49. Reda AA, Kotz D, Kocks JW, Wesseling G, van Schayck CP. Reliability and validity of the clinical COPD questionniare and chronic respiratory questionnaire. Respir Med. 2010;104(11):1675-82.

50. Patel AS, Siegert RJ, Brignall K, Gordon P, Steer S, Desai SR, et al. The development and validation of the King's Brief Interstitial Lung Disease (KBILD) health status questionnaire. Thorax. 2012;67(9):804-10.

51. Sudore RL, Stewart AL, Knight SJ, McMahan RD, Feuz M, Miao Y, et al. Development and validation of a questionnaire to detect behavior change in multiple advance care planning behaviors. PLoS One. 2013;8(9):e72465.

52. Sudore RL, Heyland DK, Barnes DE, Howard M, Fassbender K, Robinson CA, et al. Measuring advance care planning: optimizing the Advance Care Planning Engagement Survey. J Pain Symptom Manag. 2017;53(4):669-81 e8.

53. Teno JM, Fisher ES, Hamel MB, Coppola K, Dawson NV. Medical care inconsistent with patients' treatment goals: association with 1-year Medicare resource use and survival. J Am Geriatr Soc. 2002;50(3):496-500.
54. Damschroder LJ, Aron DC, Keith RE, Kirsh SR, Alexander JA, Lowery JC. Fostering implementation of health services research findings into practice: a consolidated framework for advancing implementation science. Implement Sci. 2009;4:50

55. Cella D, Eton DT, Lai JS, Peterman AH, Merkel DE. Combining anchor and distribution-based methods to derive minimal clinically important differences on the Functional Assessment of Cancer Therapy (FACT) anemia and fatigue scales. J Pain Symptom Manag. 2002;24(6):547-61.

56. Tan WC, Bourbeau J, Aaron SD, Zhou G, Maltais F, Hernandez P, et al. Global Initiative for Chronic Obstructive Lung Disease 2017 Classification and Lung Function Decline in Chronic Obstructive Pulmonary Disease. Am J Respir Crit Care Med. 2018;197(5):670-3.

\section{Ready to submit your research? Choose BMC and benefit from:}

- fast, convenient online submission

- thorough peer review by experienced researchers in your field

- rapid publication on acceptance

- support for research data, including large and complex data types

- gold Open Access which fosters wider collaboration and increased citations

- maximum visibility for your research: over $100 \mathrm{M}$ website views per year

At BMC, research is always in progress.

Learn more biomedcentral.com/submissions 